\title{
Desgaste lubricado de recubrimientos NiCrBSi refundidos parcialmente con láser ${ }^{(*)}$
}

\author{
R. Vijande*, J.M. Cuetos*, J.L. Cortizo*, E. Rodríguez* y Á. Noriega*
}

\begin{abstract}
Resumen
Los recubrimientos base níquel proyectados por plasma son ampliamente utilizados en la industria debido a su buen comportamiento a desgaste. La refusión por láser de estos recubrimientos elimina o disminuye fuertemente su porosidad, aumenta su microdureza y adherencia con el substrato. En este trabajo se define el "mallado láser" como la refusión parcial de la superficie de una capa y se aplica, para lograr unos mejores resultados antidesgaste a un recubrimiento $\mathrm{NiCrBSi}$, combinando las ventajas de la proyección por plasma y de la refusión por láser. Los resultados se verifican experimentalmente y se cuantifican atendiendo a las variables porcentaje de superficie refundida y ángulo del "cordón de mallado" (huella de refusión de los sucesivos barridos paralelos del haz del láser). El proceso experimental se desarrolla siguiendo la metodología de diseño de experimentos, para optimizar tanto el proceso de ensayos como la función objetivo de mínimo desgaste en contacto lubricado.
\end{abstract}

Palabras clave

Recubrimientos base níquel; Plasma; Refusión láser; Optimización desgaste lubricado.

\section{Lubricated wear of NiCrBSi coatings partially remelted with laser}

Abstract

Keywords

\begin{abstract}
Plasma sprayed nickel based coatings are widely used at the industry due to their good wear behaviour. The laser remelting of these coatings eliminates or strongly decreases their porosity and increases their microhardness and adherence with the substrate. In this work, we define the "laser meshing" as the partial refusion of the coating's surface. This technique is applied to a $\mathrm{NiCrBSi}$ coating in order to achieve better anti-wear outcomes, combining the advantages of plasma spraying and laser remelting. The results are experimentally verificated and they quantify themselves attending to the variables percentage of remelted surface and angle of "meshing cord" (remelting trace of successive parallel tracks by a laser beam). The experimental process is developed following the DOE methodology, to optimize both the test process and the objective function of minimum wear in lubricated contact.
\end{abstract}

Nickel based coatings; Plasma; Laser remelting; Lubricated wear optimization.

\section{INTRODUCCIÓN}

Los recubrimientos de base níquel son ampliamente utilizados en la industria debido a su buen comportamiento a desgaste, siendo la técnica de proyección por plasma una de las más utilizadas para la obtención de estos recubrimientos ${ }^{[1-4]}$. No obstante, algunas de las características que presentan estos recubrimientos proyectados por plasma, como la porosidad, microdureza y adherencia, pueden ser mejoradas empleando técnicas como la proyección al vacio o como la de fusión posterior con láser, que denominaremos refusión ${ }^{[5 \text { y } 6]}$, ya que el material del recubrimiento se funde por primera vez cuando se realiza la proyección por plasma.
En los últimos años, la técnica de refusión con láser se ha utilizado para refundir capas proyectadas por plasma, mediante sucesivas pasadas solapadas con el haz de láser sobre la superficie del recubrimiento de plasma. Con ello se consiguen los siguientes resultados: reducir la porosidad de los recubrimientos de base níquel, que pueden pasar del, aproximadamente, $3 \%$ en los recubrimientos proyectados por plasma, a eliminarse prácticamente después de la refusión con láser, aumentar la microdureza, incrementar la adherencia entre recubrimiento y substrato y homogeneizar la microestructura del recubrimiento ${ }^{[7 \text { y } 8]}$.

El comportamiento a desgaste que presentan las capas de base níquel proyectadas por plasma y refundidas posteriormente con láser, es bastante bueno

\footnotetext{
(•) Trabajo recibido el día 28 de julio de 2008 y aceptado en su forma final el día 14 de noviembre de 2008.

* Universidad de Oviedo. Área de Ingeniería Mecánica, Campus de Viesques. Edificio Departamental Oeste. Módulo 5, 33203 Gijón. Asturias.
} 
para las condiciones de contacto seco, como consecuencia de las mejoras de las características del recubrimiento antes comentadas ${ }^{[9}$ 10]. No obstante, la refusión de toda la superficie del recubrimiento con el láser presenta tres inconvenientes importantes:

En primer lugar, el tiempo necesario para realizar el proceso de refusión es elevado, lo que incrementa mucho los costes del mismo.

En segundo lugar, la eliminación de la porosidad, que produce la fusión con láser, provoca que el comportamiento a desgaste lubricado de las capas proyectadas por plasma empeore sensiblemente con respecto al que presenta la capa sin refundir, debido a que los poros que actúan como depósitos de lubricantes se reducen considerablemente o incluso se eliminan, lo que hace que exista menos lubricante en la zona de contacto y, por tanto, empeoren las condiciones de lubricación en dicha zona ${ }^{[11]}$.

En tercer lugar, debido al excesivo tiempo que el láser debe estar actuando sobre la superficie de recubrimiento a refundir, sobre todo si la superficie a tratar es grande, se produce una excesiva afectación térmica, que se traduce en tensiones internas en el recubrimiento que pueden dar lugar a un agrietamiento del mismo ${ }^{[12]}$.

Una posibilidad que permite combinar las propiedades de los recubrimientos proyectados por plasma (fundamentalmente la elevada porosidad para contactos lubricados) con las ventajas que origina el proceso de refusión con láser, es realizar sucesivos barridos paralelos con el láser, separados entre si, sobre la superficie del recubrimiento, de modo que no se refunda el $100 \%$ de la superficie del mismo y, por lo tanto, queden zonas refundidas y zonas sin refundir. A esta técnica la denominamos "mallado láser".

En este trabajo se va a estudiar el comportamiento a desgaste de un recubrimiento de base níquel, sometido a esta técnica, para un contacto lubricado con unas condiciones de carga y velocidad constantes.

\section{PROCEDIMIENTO EXPERIMENTAL}

\subsection{Recubrimiento proyectado por plasma}

Se utilizaron polvos de $66 \mu \mathrm{m}$ de diámetro medio de la aleación Eutalloy PE3309, con una composición de \% en peso: $15,7, \mathrm{Cr} ; 3,35, \mathrm{~B} ; 4,27, \mathrm{Si}$; 4,08, B; $0,81, \mathrm{C}$; bal Ni, que se pueden apreciar en la figura 1 . La proyección se realizó con un equipo de plasma de la casa Metco, utilizando como gas primario $\mathrm{N}_{2}$ con un caudal de $75 \mathrm{l} / \mathrm{min}$ y como gas secundario $\mathrm{H}_{2}$, con un caudal de $15 \mathrm{l} / \mathrm{min}$; la presión de ambos gases fue de 3,4 bares, el caudal de polvo $8 \mathrm{Kg} / \mathrm{h}$ y la distancia de proyección $100 \mathrm{~mm}$.

En estas condiciones se obtuvo un recubrimiento de la aleación $\mathrm{NiCrBSi}$ de 0,5 mm de espesor, depositado sobre un substrato de acero S355, según norma EN10027-1, con forma cilíndrica (50 mm de diámetro exterior y $25 \mathrm{~mm}$ de longitud), cuyo esquema se muestra en la figura 2 , siendo la rugosidad medía de la capa proyectada de $\mathrm{Ra}=3,39 \mu \mathrm{m}$.

Antes de realizar la refusión con láser se procedió a mecanizar la superficie del cilindro hasta dejarlo con una rugosidad media de $\mathrm{Ra}=1,39 \mu \mathrm{m}$ y un espesor de capa de 0,4 $\mathrm{mm}$.

\subsection{Refusión con láser}

Se realizó empleando un láser de $\mathrm{CO}_{2}$ de $1.700 \mathrm{~W}$ de potencia nominal que proporcionó un TEM 01*. Se buscaron los parámetros óptimos del láser que permitiesen refundir la capa, de modo que la porosidad quedase reducida casi a cero, aumentase la dureza del recubrimiento y garantizase entre recubrimiento y substrato una dilución mínima, pero suficiente para mejorar la adherencia entre ambos.

El estudio del proceso de refusión con láser, la microestructura y las características del recubrimiento antes y después del proceso de refusión con láser, se estudiaron en un trabajo previo ${ }^{[13]}$.

Una vez determinados los parámetros óptimos que se indican en la tabla I, se procedió a realizar la operación de mallado, para lo cual se realizaron barridos paralelos sucesivos con el láser, separados entre si, sobre la superficie del recubrimiento, manteniendo una

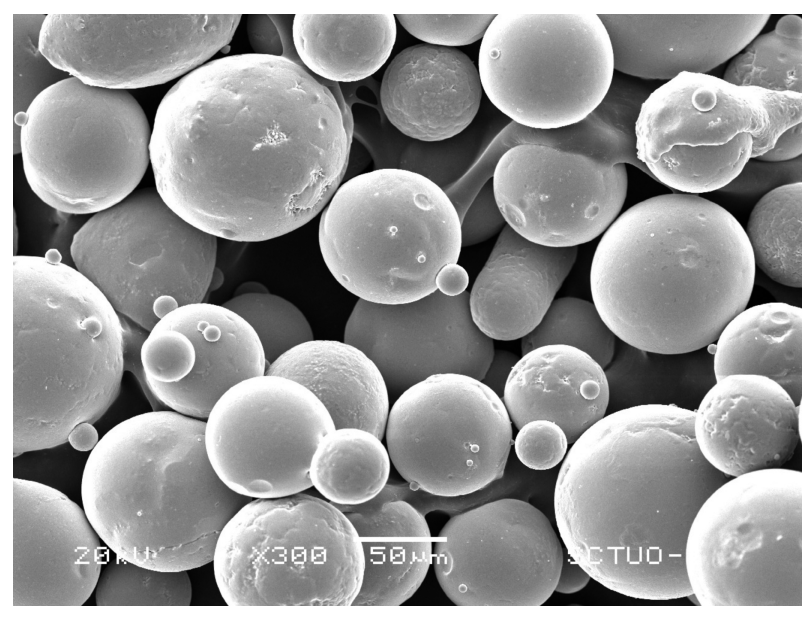

Figura 1. Micrografía SEM de los polvos de la aleación NiCrBSi.

Figure 1. SEM image of NiCrBSi in powdered form. 

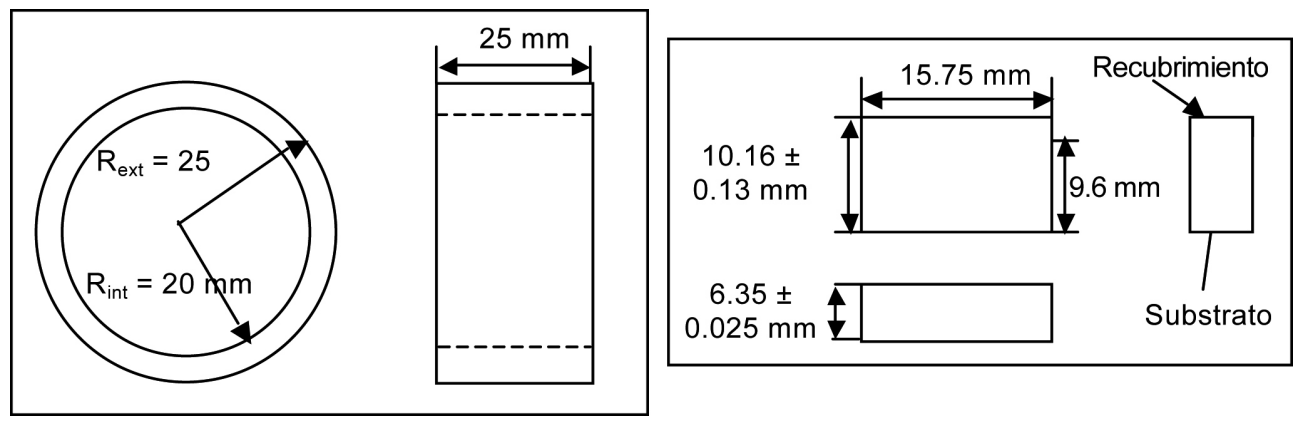

Figura 2. Dimensiones de las probetas tipo anillo y bloque.

Figure 2. Dimensions of block and ring test specimens.

Tabla I. Parámetros de láser empleados para refundir el recubrimiento base Ni proyectado por plasma

\section{Table I. Laser parameters used to refuse the} plasma sprayed Ni based coating

\begin{tabular}{lc}
\hline \multicolumn{1}{c}{ POTENCIA } & $1260 \mathrm{~W}$ \\
\hline VELOCIDAD DE BARRIDO & $300 \mathrm{~mm} / \mathrm{min}$ \\
DENSIDAD DE ENERGIA & $15.95 \mathrm{~J} / \mathrm{cm}^{2}$ \\
Focalización & $10 \mathrm{~mm}$ \\
Ancho del cordón & $1,58 \mathrm{~mm}$ \\
Gas de arrastre & $\mathrm{Ar}$ \\
Presión de gas de arrastre & $2 \mathrm{bar}$ \\
Presión de gas cabezal & $0,5 \mathrm{bar}$ \\
\hline
\end{tabular}

distancia focal fija que permitió obtener franjas de superficie refundida (cordones) de 1,58 $\mathrm{mm}$ de ancho.
En diferentes probetas se fue variando la separación entre los cordones, lo que permitió ir obteniendo porcentajes de superficie refundida del $12.5 \%, 25 \%$, $37,5 \%$ y $50 \%$ del total de la superficie del recubrimiento. Se realizaron otros ensayos para porcentajes de refusión superiores al $50 \%$, observándose que la superficie afectada por los efectos térmicos del láser abarcaba casi el total de la superficie del anillo. También se prepararon anillos con el $100 \%$ de superficie refundida, con objeto de contrastar resultados.

Igualmente, se fue variando el ángulo que formaba la dirección de desplazamiento del láser con respecto a la base del anillo desde 0 a $90^{\circ}$, en intervalos de $22,5^{\circ}$.

En la figura 3 se muestran diferentes geometrías de mallado obtenidas.

Con el objeto de dar un mejor acabado final, se realizó un nuevo mecanizado de la superficie después de la refusión con láser, de modo que la capa quedó finalmente con un espesor medio de $0,36 \mathrm{~mm}$ y el mismo Ra anterior de 1,39 $\mu$ m en la zona sin refun-

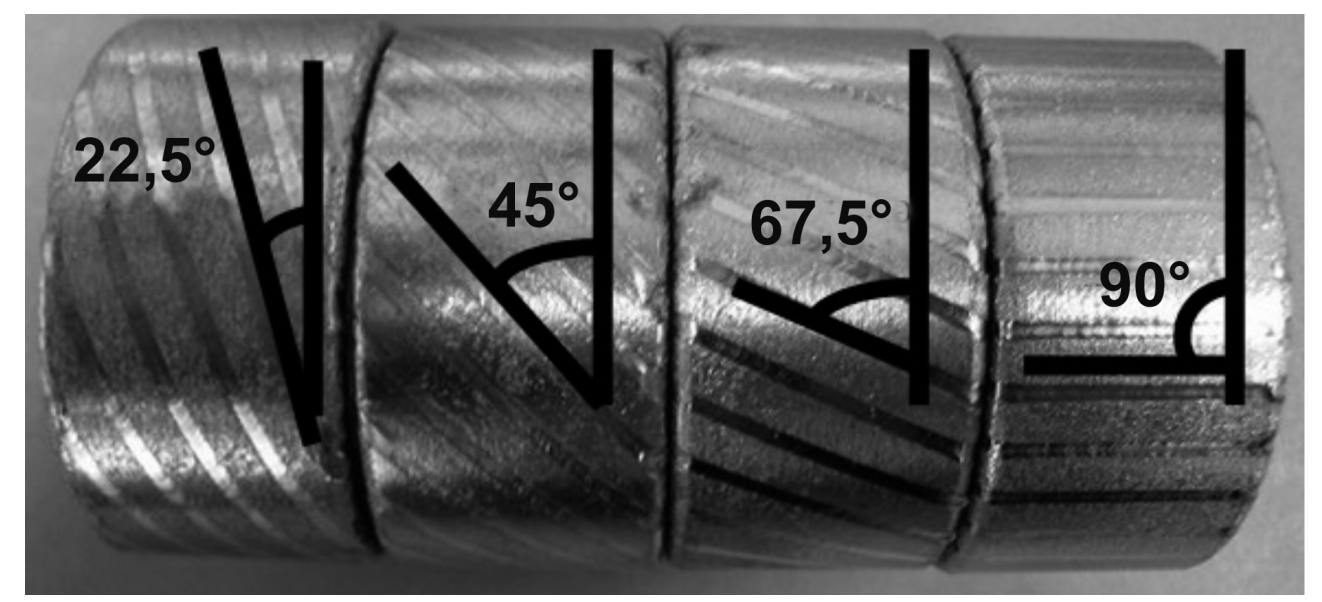

Figura 3. Diferentes geometrías de mallado láser.

Figure 3. Different geometries of "laser meshing". 
dir con láser y una rugosidad menor $(\mathrm{Ra}=0,56 \mu \mathrm{m})$, en la zona refundida.

\subsection{Proceso de desgaste}

Los ensayos de desgaste se realizaron siguiendo la norma ASTM G77, para un contacto de deslizamiento lubricado bloque-anillo. El anillo empleado es el del recubrimiento mallado, estando el bloque constituido por un substrato de acero y un recubrimiento de WC depositado por la técnica de laser cladding (Fig. 2), que permitió obtener un recubrimiento de una dureza media de $1.250 \mathrm{HV}_{300}$ que garantizase un desgaste mínimo del anillo para la condición de lubricado.

La carga utilizada ha sido de $377,3 \mathrm{~N}$ y la velocidad de deslizamiento de $0,68 \mathrm{~m} / \mathrm{s}$, con una duración de los ensayos de 100.000 ciclos, que se corresponden con una distancia deslizada de $16.368 \mathrm{~m}$.

Como lubricante se utilizó uno suministrado por Repsol, que contiene un anticorrosivo y modificador de fricción "Mid AW" con unas viscosidades de $43,87 \mathrm{~mm} / \mathrm{s}^{2}$ a $40^{\circ} \mathrm{C}$ y $6,481 \mathrm{~mm} / \mathrm{s}^{2}$ a $100{ }^{\circ} \mathrm{C}$, según la norma ASTM D445 y un índice de viscosidad de 96 según la norma ASTM D2270.

Para la planificación de los ensayos de desgaste, teniendo en cuenta la experiencia del grupo de trabajo $^{[14-16]}$, se empleó la metodología de diseño de experimentos, usando el software Statgraphics Centgurion XV, bajo las condiciones de carga y velocidad constantes antes indicadas. Se seleccionó un diseño de tipo multinivel factorial en cuatro niveles, lo que da un total de 16 ensayos, tomando como factores experimentales el porcentaje de superficie del anillo refundida y el ángulo de mallado.

Tabla II. Valores introducidos en el software Statgraphics Centurión XV para los factores experimentales porcentaje de superficie refundida y ángulo de mallado

Table II. Values used in the software Statgraphics Centurión XV to the experimental factors percentage of remelted surface and laser meshing

\begin{tabular}{cc}
\hline $\begin{array}{c}\text { \% Superficie } \\
\text { refundida }\end{array}$ & $\begin{array}{c}\text { Ángulo de } \\
\text { mallado en grados }\end{array}$ \\
\hline 12,5 & 22,5 \\
25 & 45 \\
37,5 & 67,5 \\
50 & 90 \\
\hline
\end{tabular}

Cada ensayo de desgaste se repitió tres veces, con el fin de valorar la variabilidad del proceso.

En la tabla II se muestran los valores introducidos para los dos factores experimentales.

\section{RESULTADOSY DISCUSIÓN}

\subsection{Propiedades del recubrimiento}

Las características de este recubrimiento han sido estudiadas por los autores en un trabajo previo ${ }^{[17]}$.

La dureza Vickers del recubrimiento pasó de los 900 $\mathrm{HV}_{300}$ de dureza media en la zona sin refundir, a durezas medias de $1.150 \mathrm{HV}_{300}$ en la zona refundida, apreciándose además una transición más suave de la dureza entre recubrimiento y substrato en la zona refundida, como consecuencia de la dilución alcanzada entre recubrimiento y substrato en dicha zona.

La figura 4 muestra la sección transversal del recubrimiento, antes (Fig. 4a)) y después (Fig. 4b)) de la refusión con láser, donde se aprecia el cambio de la porosidad, determinada mediante microscopía óptica, que pasa de un valor medio de $3.75 \%$ en la zona sin refundir a un valor de prácticamente 0 en la zona refundida.

En cuanto a los valores de adherencia del recubrimiento, la refusión con láser origina una mejora de la misma, que va aumentando al incrementar el porcentaje de superficie refundida, debido a la dilución entre recubrimiento y substrato en la zona de interfase, oscilando entre los $17 \mathrm{MPa}$ de la superficie sin refundir y los $40 \mathrm{MPa}$ de superficie completamente refundida con láser.

\subsection{Desgaste}

Los valores de desgaste obtenidos en cada caso, una vez que el software determinase la aleatoriedad en el orden de entrada, se muestran en la tabla III.

Además de esos ensayos considerados en el diseño de experimentos, se realizaron también los ensayos con el $100 \%$ de la superficie del anillo refundida y con la superficie del anillo sin mallar (0\%). Los resultados obtenidos han sido de $21,80 \mathrm{mg}$ de desgaste para el $100 \%$ del porcentaje de superficie refundida y de 68,60 mg para la superficie sin mallar.

Se realizó un análisis de varianza (Anova) de los datos experimentales obtenidos, cuyos resultados se reflejaron mediante el diagrama de Pareto para el desgaste del anillo mallado, que se muestra en la figura 5. En este diagrama se puede apreciar la influencia, en orden decreciente, de cada uno de los factores 

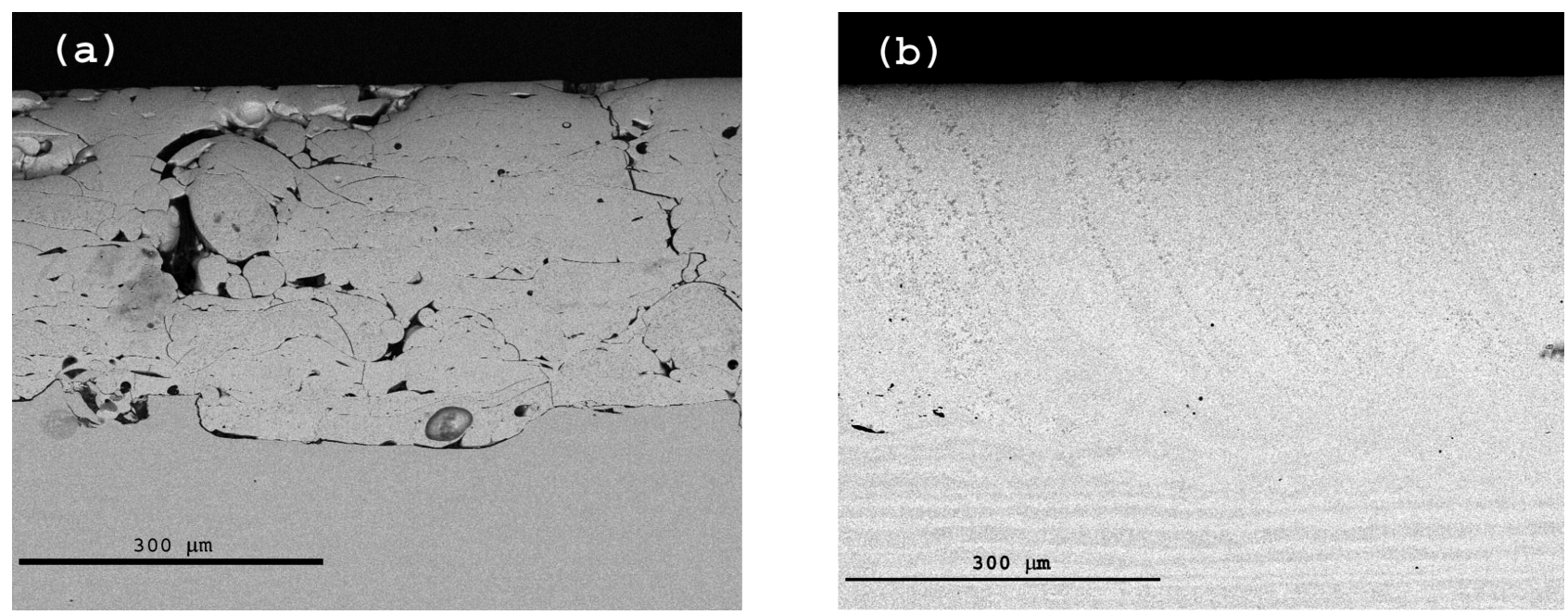

Figura 4. Sección transversal del recubrimiento, antes (Fig. 4a)) y después (Fig. 4b)), de la refusión con láser.

Figure 4. Cross section of coating, before (Fig. 4a)) and after (Fig. 4b)), laser remelting.

Tabla III. Valores medios de desgaste obtenidos en función del porcentaje de superficie refundida y del ángulo de mallado, en el orden aleatorio generado por el software Statgraphics Centurión XV

Table III. Mean wear values obtained in function of the percentage of remelted surface and the angle of laser meshing, in the random order generated by the software Statgraphics Centurión XV

\begin{tabular}{cll}
\hline $\begin{array}{c}\text { \% S } \\
\text { refundida }\end{array}$ & $\begin{array}{c}\text { Angulo } \\
\text { mallado }\end{array}$ & $\begin{array}{c}\text { Desgaste } \\
(\mathbf{m g})\end{array}$ \\
\hline 37,5 & 67,5 & 48,1 \\
50 & 45 & 32,1 \\
12,5 & 45 & 50,35 \\
12,5 & 22,5 & 54,3 \\
25 & 67,5 & 52,25 \\
25 & 45 & 47,6 \\
50 & 22,5 & 29,65 \\
12,5 & 67,5 & 66 \\
12,5 & 90 & 62,5 \\
25 & 90 & 53,45 \\
50 & 90 & 39,1 \\
37,5 & 22,5 & 36,3 \\
37,5 & 45 & 35,2 \\
25 & 22,5 & 48,6 \\
50 & 67,5 & 39,6 \\
37,5 & 90 & 49,8 \\
\hline
\end{tabular}

experimentales (\% superficie refundida, ángulo de mallado) y las interacciones entre ellos en la variabilidad de la respuesta.

En la tabla IV se muestra la variabilidad en el desgaste de forma separada para cada uno de los factores experimentales. La significación estadística de cada uno de los factores experimentales se prueba comparando la media de $\mathrm{R}$ cuadrado $\left(\mathrm{R}^{2}\right)$ con la estimación del error experimental. En este caso, solamente los dos primeros efectos, porcentaje de superficie mallado y ángulo de mallado presentan "valores $\mathrm{P}$ " inferiores a 0,05 , indicando que son significativamente diferentes de cero para un nivel de confianza del $95 \%$.

A la vista del poco significado que presentan los efectos porcentaje de superficie refundida al cuadrado (AA), porcentaje de superficie refundida por ángulo de mallado $(\mathrm{AB})$ y ángulo de mallado al cuadrado (BB) se realizó un nuevo ajuste del estudio eliminando esos efectos poco significativos.

La figura 6 muestra un nuevo diagrama de Pareto obtenido una vez optimizado, eliminando del análisis los efectos no significantes de los factores experimentales. Igualmente, se aprecia que el desgaste es directamente proporcional al valor del ángulo de mallado (representado por el signo + en la figura) e inversamente proporcional al valor del porcentaje de superficie refundida (signo - en la figura), siendo el factor experimental más significativo sobre el desgaste el porcentaje de superficie refundida.

En la tabla $V$ se muestran los resultados finales de la variabilidad en el desgaste, de forma separada para cada uno de los factores experimentales que 


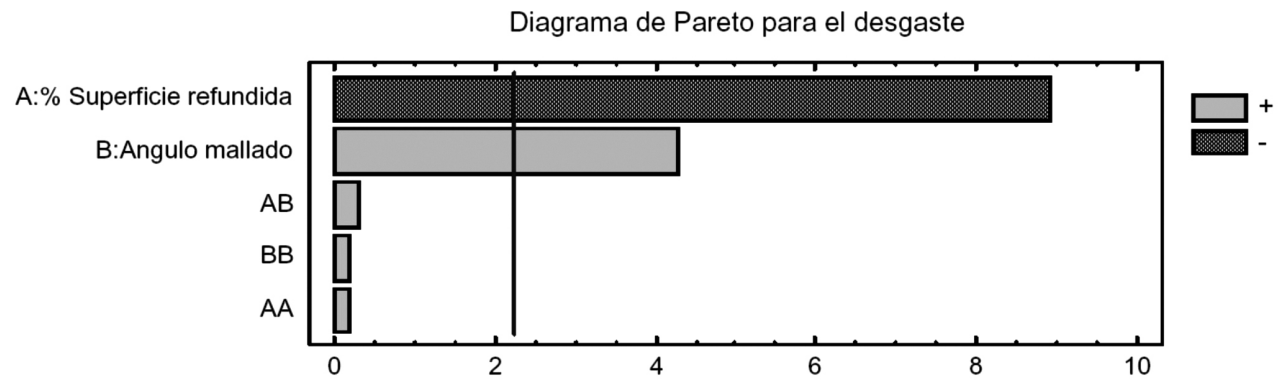

Figura 5. Diagrama de Pareto para el desgaste del anillo mallado.

Figure 5. Pareto's diagram for the wear of the ring meshed.

Tabla IV. Variabilidad obtenida en el desgaste de forma separada para cada uno de los dos factores experimentales considerados

Table IV. Obtained variability of wear in separated form for both of two experimental factors considered

\begin{tabular}{ll}
\hline \multicolumn{1}{c}{ Fuente } & P-Valor \\
\hline A:\% S refundida & 0,0000 \\
B:Angulo mallado & 0,0017 \\
AA & 0,8863 \\
AB & 0,7942 \\
BB & 0,8765 \\
\hline
\end{tabular}

finalmente han quedado como más significativos (con "valores P" inferiores a 0,05).

El estadístico R cuadrado indica que el modelo así ajustado explica el 90,51 \% de la variabilidad en
Tabla V. Resultados finales de la variabilidad obtenida en el desgaste una vez eliminados los efectos no significantes de los factores experimentales

Table V. Final results of variability obtained in wear once no significant effects have been eliminated of experimental factors

\begin{tabular}{ll}
\hline \multicolumn{1}{c}{ F-Ratio } & $\boldsymbol{P}$-Valor \\
\hline A:\% S refundida & 0,0000 \\
B:Angulo mallado & 0,0003 \\
\hline
\end{tabular}

R-cuadrado $=90,51 \%$

R-cuadrado (ajustado para d.f.) $=89,05 \%$

Error estandar de est. $=3,46$

Error medio absoluto $=2,45$

Estadístico Durbin-Watson $=1,997(P=0,449)$

Autocorrelación residual $=-0,047$

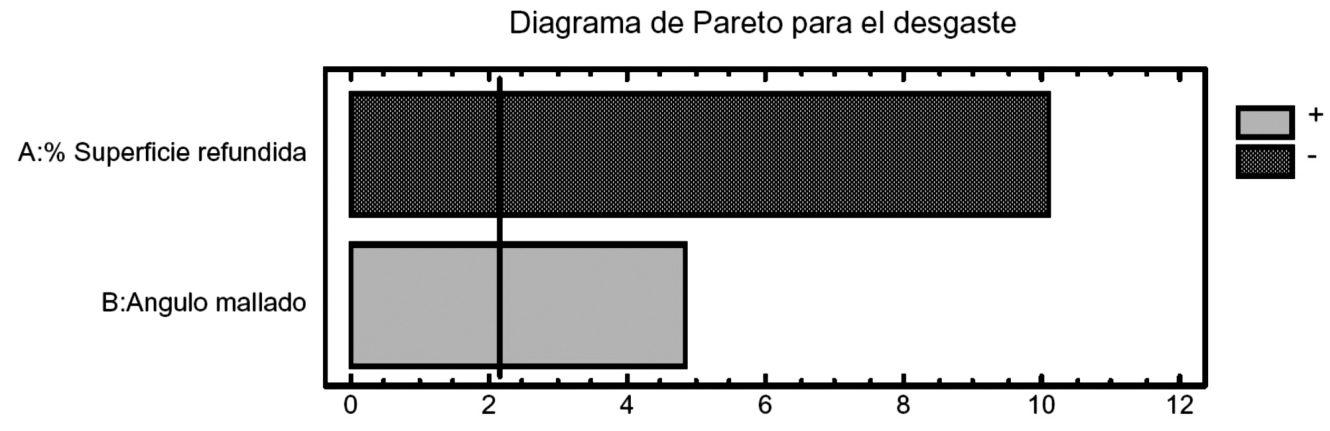

Figura 6. Diagrama de Pareto optimizado, una vez eliminados del análisis los efectos no significantes de los factores experimentales.

Figure 6. Optimized Pareto's diagram, once no significant effects of experimental factors have been eliminated from the analysis. 
el desgaste, siendo el R cuadrado ajustado 89,05 \%. El error estandar de la estimación, cuyo valor es 3,46 muestra la desviación normal de los residuos, mientras que el error absoluto de la media, que promedia el valor de los residuos, es de 1,997. El estadístico Durban Watson examina los residuos para determinar si hay cualquier correlación significativa basada en el orden en el que se suceden en el fichero de datos; dado que el $\mathrm{P}$-valor es 0,449 (superior a 0,05), no existen indicios de autocorrelación en los residuos.

La ecuación del modelo ajustado para el desgaste es:

$$
\begin{gathered}
\text { Desgaste }=56,675-0,6212 * \% \text { S refundida } \\
+0,165222 \text { *ángulo mallado }
\end{gathered}
$$

Donde los valores de las variables están especificados en sus unidades originales, es decir, desgaste en mg, superficie refundida en \% del total y ángulo de mallado en grados.

La figura 7 muestra el gráfico de efectos principales para los dos factores experimentales considerados. En dicho gráfico se aprecian variaciones lineales de la variable respuesta (desgaste), para cada uno de los dos factores experimentales contemplados. Se aprecia que el desgaste disminuye al aumentar el porcentaje de superficie refundida y al disminuir el ángulo de mallado.

La respuesta óptima que se buscó fue la de una geometría que combinase el porcentaje de superficie refundida y el ángulo de mallado de modo que se minimizase el desgaste producido en el anillo. Para ello se empleó una "función de conveniencia" de "minimización" de desgaste.

En la figura 8 se ve la superficie de respuesta obtenida para los valores del desgaste en el anillo en

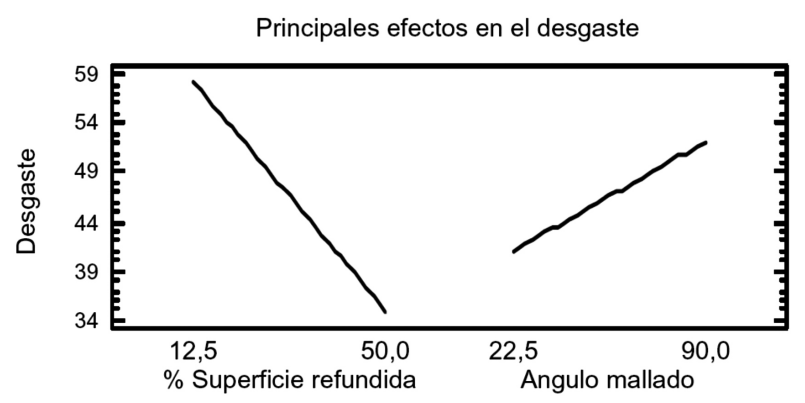

Figura 7. Gráfico de efectos principales para los dos factores experimentales considerados, ángulo y porcentaje de superficie refundida.

Figure 7. Graphic of main effects for the two experimental factors considered, angle and percentage of remelted surface.

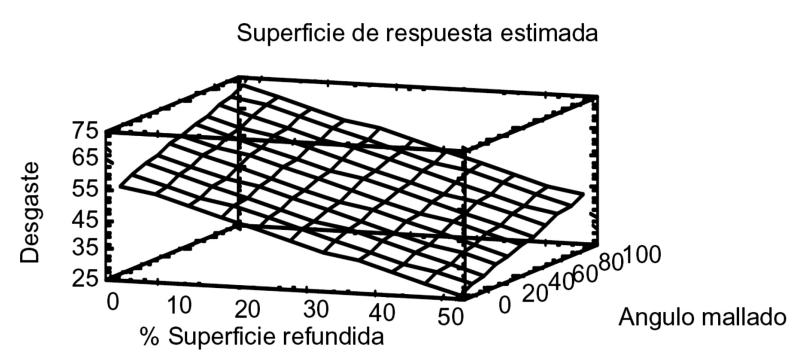

Figura 8. Superficie de respuesta obtenida para los valores del desgaste en el anillo en función de los dos factores experimentales estudiados, ángulo y porcentaje de superficie refundida.

Figure 8. Response surface obtained for the wear results of the ring in terms of the two experimental factors studied, angle and percentage of remelted surface.

Tabla VI. Valores del porcentaje de superficie refundida y del ángulo de mallado para lograr la minimización del desgaste y valor de la optimización alcanzada

Table VI. Values of percentage of remelted surface and meshing angle to achieve the minimization of wear and value of attained optimization

Valor óptimo $=0,991014$

\begin{tabular}{cccc}
\hline $\begin{array}{c}\text { Factor } \\
\text { experimental }\end{array}$ & Bajo & Alto & Optimo \\
\hline \% S refundida & 12,5 & 50,0 & 50,0 \\
Angulo mallado & 22,5 & 90,0 & 22,5 \\
\hline Respuesta & & Optimo \\
\hline Desgaste & & 29,3325 \\
\hline
\end{tabular}

función de los dos factores experimentales estudiados, ángulo y porcentaje de superficie refundida.

En la tabla VI se muestran los valores del porcentaje de superficie refundida y del ángulo de mallado para lograr la minimización del desgaste, mostrándose igualmente el grado de optimización alcanzado, 0,992721 sobre 1 . Se aprecia que la citada minimización del desgaste se produce para unos valores del $50 \%$ de porcentaje de superficie refundida y un ángulo de mallado de $22,5^{\circ}$, correspondiendo para estos 


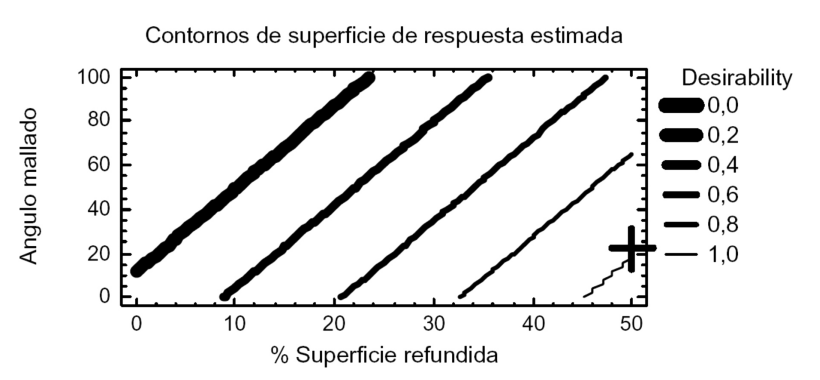

a)

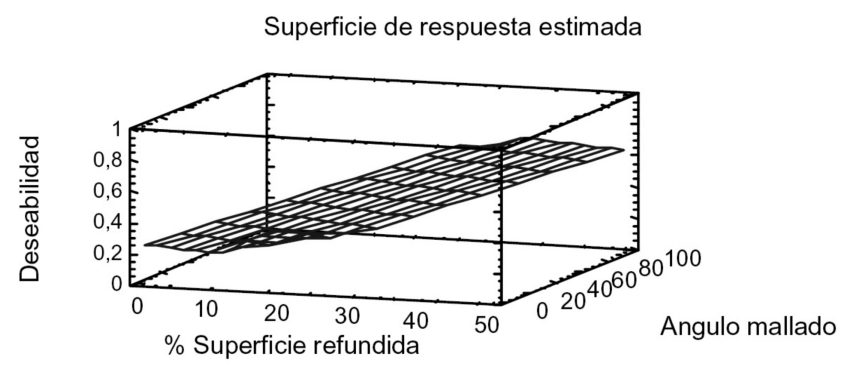

b)

Figura 9. a) Contornos y b) curva de respuesta estimada, para los dos factores experimentales estudiados en función del grado de optimización alcanzado.

\section{Figure 9. a) Contours and b) response curve estimated, for the two experimental factors considered in terms of grade of optimization attained.}

parámetros un desgaste de 29,33 mg, que se encuentra muy próximo al valor experimental obtenido para estos parámetros, que ha sido de 29,65 mg.

En las figuras 9 a) y b) se muestran, respectivamente, los contornos y la curva y de respuesta estimada, respectivamente, para los dos factores experimentales estudiados en función del grado de optimización alcanzado.

\subsection{Justificación de la influencia del porcentaje de superficie refundida y del ángulo de mallado}

El porcentaje de superficie refundida se estudió entre los valores de $0 \%$ (superficie sin refundir con láser) y $50 \%$ de superficie refundida. Para valores superiores al $50 \%$ de superficie refundida el valor del desgaste presenta una disminución muy reducida. En valor absoluto, pasaría de los $29,33 \cdot 10^{-4} \mathrm{~g}$ ( $50 \%$ de superficie refundida con un ángulo de $22,5^{\circ}$ ), a los $21,80 \cdot 10^{-4} \mathrm{~g}$ que correspondería a una superficie refundida del $100 \%$ y el mismo ángulo.

La refusión del $100 \%$ del porcentaje de la superficie del recubrimiento llevaría aparejado, además de un coste mucho más elevado, el problema de optimizar de nuevo los parámetros del láser, debido al necesario solapamiento entre los cordones de refusión.

Un valor de superficie refundida por encima del 50 $\%$ implicaría que las zonas entre cordones se empiezan a ver afectadas térmicamente por el proceso de refusión con láser, con lo que prácticamente toda la superficie estaría afectada. Además, ello daría lugar a un incremento muy elevado del tiempo de tratamiento con láser de la superficie, lo que haría que el coste del mismo aumentase considerablemente.
Todo ello hace que la refusión del $50 \%$ de la superficie, para las condiciones de carga y velocidad estudiadas, se considere lo más efectivo, teniendo en cuenta aspectos técnicos y económicos conjuntamente.

En cuanto al efecto del ángulo de mallado, se aprecia como a medida que se incrementa éste, el valor del desgaste aumenta (Fig. 7).

Ángulos de mallado próximos a los cero grados implicarían cordones casi paralelos a la dirección de deslizamiento, con lo cual habría una parte del contacto en el que la superficie estaría siempre refundida y otra parte que estaría siempre sin refundir. Ello daría lugar a concentraciones de desgaste altas en la zona sin refundir, mientras que en la zona refundida, al no haber depósito de lubricante (falta de porosidad y no arrastre de lubricante de zonas sin refundir) el desgaste también sería más elevado. Ello hace que no se consideren ángulos de mallado muy bajos (por debajo de $22,5^{\circ}$ ).

Con el fin de explicar esta influencia del valor del ángulo de mallado, se realizaron cálculos con el software "matlab", que permitieron interpretar la evolución del valor de la superficie unitaria $(1 \mathrm{~mm}$ de ancho), de contacto entre bloque y anillo, en función de la distancia deslizada y para diferentes ángulos de mallado, de acuerdo con el esquema que se muestra en la figura 10.

La figura 11 muestra los resultados obtenidos para el porcentaje de superficie refundida del $50 \%$ y ángulos de mallado de $22,5^{\circ}, 45^{\circ}, 67,5^{\circ}$ y $90^{\circ}$.

Se puede apreciar en ella que, para un ángulo de mallado de $22,5^{\circ}$, el valor de la superficie unitaria refundida por el láser, en la zona de contacto bloqueanillo, es la que menos variaciones experimenta de los cuatro ángulos considerados. Ello implicaría que para esta geometría, en las condiciones estudiadas, 


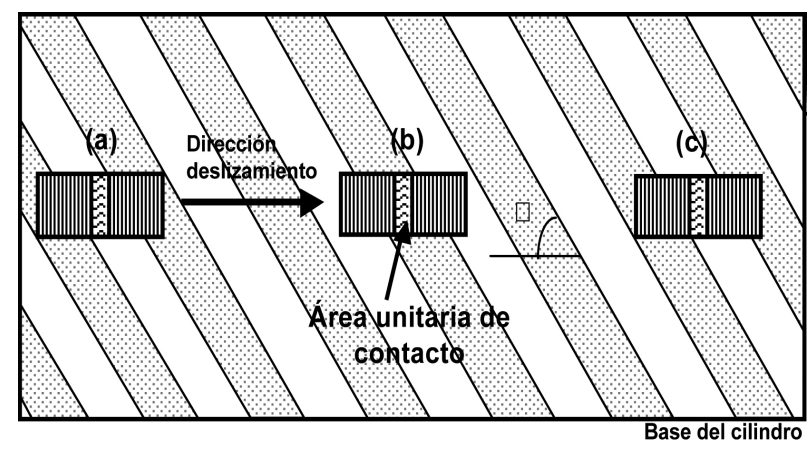

(a) Inicio del contacto entre el área unitaria y el cordón.

(b) Máxima superficie del contacto entre el área unitaria y el cordón.

(c) Fin del contacto entre el área unitaria y el cordón.

$\mathrm{a}$ = ángulo del cordón de mallado

Figura 10. Esquema del contacto del área unitaria de bloque (1 $\mathrm{mm}$ de ancho de huella), con diferentes zonas del anillo).

Figure 10. Representation of contact on unitary block's area (worn surface $1 \mathrm{~mm}$ wide), with different zones of the ring.

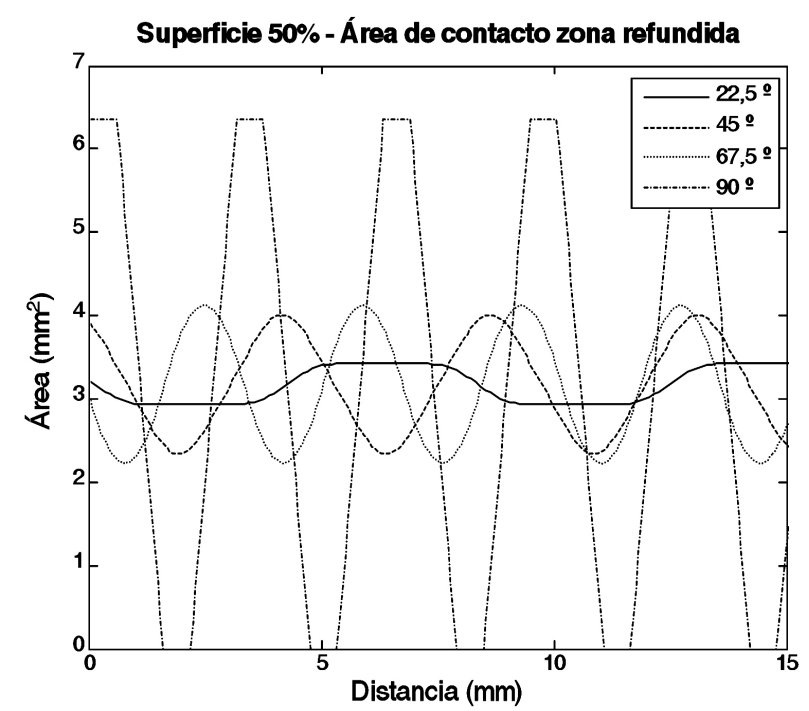

Figura 11. Valores del área de contacto unitaria con cordones de mallado, en relación con la distancia deslizada, para un $50 \%$ de superficie refundida y los cuatro ángulos de cordón considerados, $22,5^{\circ}, 45^{\circ}, 67,5$ y $90^{\circ}$

Figure 11. Values of the unitary contact area with remelted tracks, relating to the sliding distance, for a $50 \%$ of remelted surface and the four track's angles considered, $22.5^{\circ}, 45^{\circ}, 67.5^{\circ}$ and $90^{\circ}$. es para la que se produciría el mejor equilibrio entre contacto del bloque con zona refundida y con zona no refundida del anillo, sucediendo, además, que para este ángulo de $22,5^{\circ}$ es para el que en la zona de contacto se va pasando, más progresivamente, de una zona refundida a una zona no refundida, lo que originaría transiciones menos bruscas entre una zona y otra y, por ello, una mejor optimización del contacto y de las condiciones de lubricación. Al ir incrementando en ángulo de mallado se aprecia que los cambios en el valor de la superficie refundida unitaria en la zona de contacto se producen más rápidamente, lo que significaría transiciones más bruscas en el contacto y por ello incremento en el desgaste.

\subsection{Mecanismo de desgaste}

El análisis micrográfico de las superficies desgastadas del anillo indica que el mecanismo de desgaste que actúa es el abrasivo. La elevada carga para la que se realizó el ensayo $(377,3 \mathrm{~N})$, establece un régimen de lubricación límite en la zona de contacto, en el que las elevadas durezas de las partículas del recubrimiento de WC del bloque, que se enfrenta al anillo, hace que actúen como abrasivo, dando lugar al aspecto de arado en la superficie desgastada del anillo, con los surcos característicos del desgaste abrasivo, tal como se aprecia en la micrografía de la figura 12 y que coincide con los estudios de otros autores ${ }^{[18]}$.

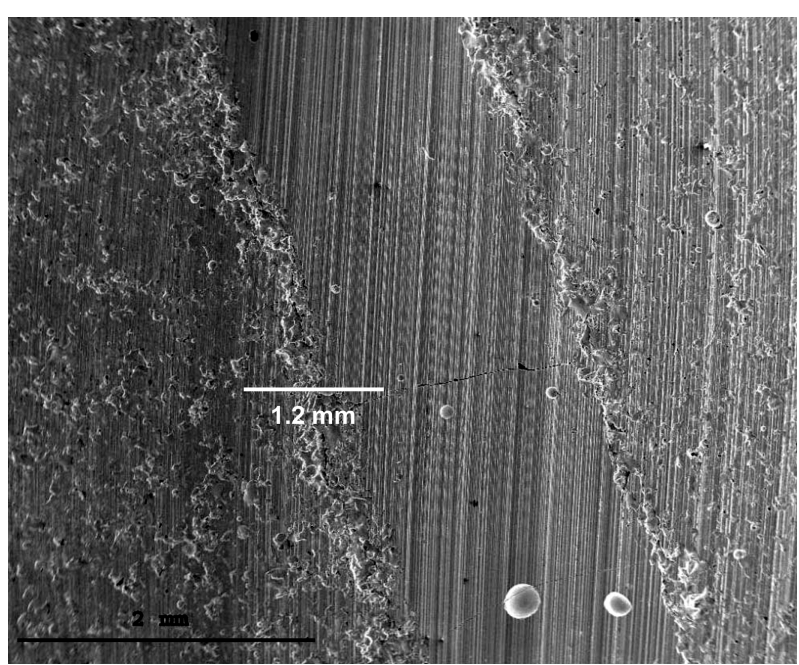

Figura 12. Imagen SEM de la huella de desgaste en que muestra una zona refundida con láser y otra zona no refundida.

Figure 12. SEM image of worn surface that shows a zone with laser remelting and another without it. 


\section{CONCLUSIONES}

Los recubrimientos de base níquel presentan un buen comportamiento a desgaste en condiciones de contacto lubricado. Este comportamiento puede ser mejorado realizando una refusión con láser de la superficie del recubrimiento, lo cual origina también un aumento de la adherencia entre recubrimiento y substrato, un incremento de la dureza del recubrimiento y una disminución de la porosidad.

Se ha definido el mallado láser como la realización de barridos paralelos con el láser, separados entre si, sobre la superficie del recubrimiento, de modo que no se refunda el $100 \%$ de la superficie del mismo y, por lo tanto, queden zonas refundidas y zonas sin refundir.

Se ha utilizado el mallado láser en recubrimientos base níquel proyectados por plasma para lograr las mejoras que producen la refusión láser.

$\mathrm{Al}$ incrementarse el porcentaje de superficie refundida con láser se va reduciendo ligeramente el desgaste producido, obteniéndose para un $50 \%$ de superficie refundida valores de desgaste muy próximos a los que se obtendrían para un $100 \%$ de superficie refundida.

$\mathrm{Al}$ ir aumentando el ángulo de mallado el valor del desgaste va aumentando, obteniéndose los menores valores para ángulos próximos a los $22,5^{\circ}$. Para ángulos próximos a $0^{\circ}$, los cordones refusión láser serían casi paralelos a la dirección de deslizamiento, por lo que existe la posibilidad de que el contacto se produzca siempre en una zona refundida o en una zona no refundida, dependiendo de las dimensiones del contacto y de los tamaños de los cordones láser.

El mecanismo de desgaste que opera es el abrasivo.

\section{Agradecimientos}

Los autores agradecen al MEC por la financiación del proyecto MAT2007/61777, en el marco del cual se ha desarrollado el presente trabajo.

\section{REFERENCIAS}

[1] V. Fridrici, S. Fouvry y P. Lapsa, Surf. Coat. Technol. 163-164 (2003) 429-434.

[2] Martín, J. Rodríguez, J. E. Fernández y R. Vijande, Wear 251 (2001) 1.017-1.022.

[3] R. Vijande, F.J. Belzunce, J.E. Fernández; M.C. Bécares y A. Rincón, Wear 148 (1995) 221-233.

[4] C. Navas, R. Vijande, J.M. Cuetos, M.R. Fernández y J. Damborenea. Surf. Coat. Technol. 201 (2006) 776-785.

[5] G. Cano, N. Cinca, S. Dosta y J. M. Guillemany, Rev. Metal. Madrid 44 (2008) 197-205.

[6] S. Schelz, N. Branland, D. Plessis, B. Minot y F. Guillet, Surf. Coat. Technol. 200 (2006) 6.3846.388.

[7] G.Y. Liang y T.T. Wong, Surf. Coat. Technol. 89 (1997) 121-126.

[8] R. Krishnan, S. Dash, R. Kesavamoorthy, C. Babu Rao y A.K. Tyagi, Surf. Coat. Technol. 200 (2006) 2.791-2.799.

[9] J.M. Cuetos, E. Fernández, R. Vijande, A. Rincón y MC. Pérez, Wear 169 (1993) 173-179.

[10] E. Fernández, J.M. Cuetos, R. Vijande y A. Rincón, Tribol. Int. 29 (1996) 477-485.

[11] E. Fernández, M. Cadenas, J.M. Cuetos y J. Mateos, Proc. of the Fourteenth Int. Conf.. on Surface Modification Technologies XIV (SMT 14), vol. 14, París, Francia, 2000, T.S. Sudarshan y M. Jeandin (Eds.), ASM International, London, Inglaterra, 2001, pp. 333-337.

[12] J. Mateos, J.M. Cuetos, E. Fernández, y R. Vijande, Wear 239 (2000) 274-281.

[13] D. Felgueroso, R. Vijande, J.M. Cuetos, R. Tucho, A. Hernández, Wear 264 (2008) 257-263.

[14] J. Mateos, J.M. Cuetos, R. Vijande y E. Fernández. Tribol. Int. 34 (2001) 345-351.

[15] R. Vijande, F.J. Belzunce, J.E. Fernández, M.C. Bécares, y A. Rincón. Wear 148 (1995) 221-233.

[16] R. González, M. Cadenas, M.R. Fernández, J.L. Cortizo y E. Rodríguez. Wear 262 (2007) 301-307.

[17] M. Cadenas, R. Vijande, H.J. Montes, J.M. Sierra, Wear 212 (1997) 244-253.

[18] H. Wang, Wear 195 (1996) 47-52. 